\title{
1 Executive summary: Introduction to the EURACT Performance Agenda
}

\section{Stefan Wilm}

General practice/family medicine is a pivotal part of primary care systems: This fact is widely recognized all over the world ${ }^{1,2,3}$, being popular with patients able to retain a personal relationship with their doctor in the increasingly impersonal world of health-care delivery, and with politicians because of its inherent cost effectiveness ${ }^{4}$. Evidence has shown that health care systems based on effective primary care provided by trained general practitioners working in the community are not only cost effective but provide effective care for patients ${ }^{5}$. This has been recognized in Europe, where, for the majority of European countries, general practice/family medicine is an important, fundamental part of the health care systems. The European Union has built general practice into its healthcare policies, and it is being developed by non-member states as well ${ }^{6}$. General practitioners play a central role in ensuring that patients acquire appropriate health care provision. Although there has been a great deal of progress in the development of general practice in Europe, there remain large differences in the way this has been done in different countries and in the manner in which family doctors are integrated into the health care system ${ }^{7}$.

A big step forward was taken in 2002 when WONCA Europe, the academic body for general practice, published the new European definition of general practice/family medicine ${ }^{8}$. This work had come about as a result of revisiting previous definitions, as it had been felt that these were outdated and needed revising for the 21 st century. At the outset it was recognised that the essential elements of the discipline of general practice/family medicine needed to be defined first, and only when this had been done could the role definition of the family doctor be derived from them.

The definition contains the eleven characteristics which are fundamental to the discipline and are generalisable to all health-care systems in all countries regardless of contextual differences. These were then combined into a role description of the family doctor.

The document does not stop there, but goes on to explore the competencies that are required to become a skilled exponent of the discipline. It describes how these characteristics can be grouped together into six core competencies, 
and how the acquisition of these can be converted into abilities every family doctor should be able to master, and eventually into actual performance ${ }^{9}$ (see chapter 2).

EURACT developed this thinking further and produced its educational agenda in 2005. EURACT, the European Academy of Teachers in General Practice/Family Medicine, has the constitutional aim to foster and maintain high standards of care in European general practice/family medicine by promoting the discipline through learning and teaching. The EURACT Educational Agen$d a^{10}$ provided a comprehensive framework to teach the core competencies being a blueprint for a common curriculum. The educational agenda is a longitudinal strategy in regard to the learning process, including basic medical education (BME), specialty or vocational training (ST), and continuing medical education (CME)/continuing professional development (CPD), but focussing on specialty training. Based on learning in a primary care setting, it sets learning aims, is based on modern educational methods and monitors progress of learners as well as the achievement of goals. It is developed for educators, curriculum builders, educational boards, policy makers and other medical specialists.

Just like the European definition, the educational agenda is supposed to be applicable regardless of differences in health care systems, educational programmes and health care policies. It aims at harmonization of learning outcomes of educational programmes in general practice/family medicine in all European countries.

General practice/family medicine is an academic and scientific discipline with its own educational content, research, evidence base and clinical activity, and a clinical specialty orientated to primary care. The discipline has a contribution to make to the education of doctors at all stages in their training. Changes in attitudes of medical learners generally occur after learners have the opportunity to grapple with primary care problems themselves and observe their teachers coping successfully with problems presented ${ }^{11}$.

At the BME level, all students ${ }^{12}$, irrespective of their future professional career choice ${ }^{13}$, should follow a curriculum, which is directed at enabling them to understand the role and specificity of primary care and general practice ${ }^{14,15}$. Early clinical exposure of medical learners for brief periods from several days to weeks at the beginning of medical school can lay the groundwork for the medical student to help make sense of all medical training ${ }^{16}$. Lectures or small group seminars in the pre-clinical period can be effective in providing some of the knowledge required before starting work with patients ${ }^{17}$. The prime peri- 


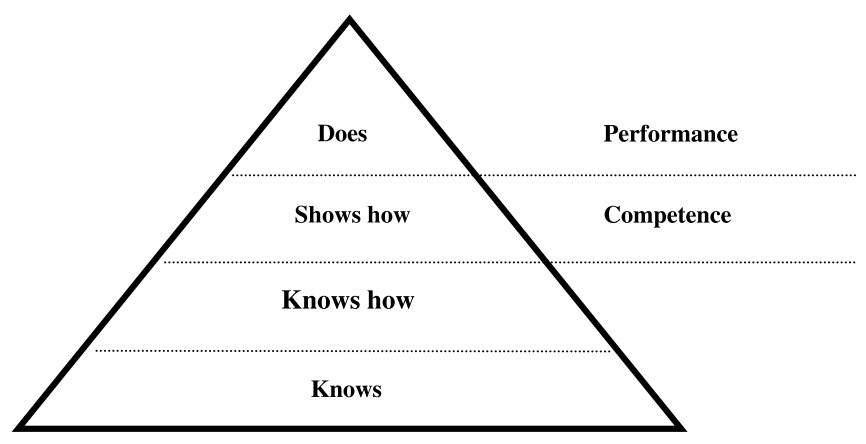

Figure 1: Miller's pyramid

od for training learners in primary care management is the student clerkship/ attachment/rotation in general practice, lasting several weeks occurring in the later years of medical school ${ }^{18,19}$.

The in-depth preparation of medical graduates to gain the competencies of family physicians takes place at the ST level. This part of medical education usually lasting several years, and in the EU a minimum of three years ${ }^{20}$, allows trainees to acquire the knowledge, skills and attitudes which are essential for safe and effective professional general practice. A large part of the programme will be based on teaching practices ${ }^{21}$.

The $C M E / C P D$ level comprises the longest phase of professional education (life-long learning). In $\mathrm{CME}$, graduated general practitioners continue to learn and change in practice in a lifelong learning scheme, thus optimizing their actual performance. CPD is understood wider as a process of planned and individually tailored learning in practice with a focus on the quality of care ${ }^{22}$. CPD may include the identification of individual learning needs, construction of a learning agenda, drawing a concrete personal learning plan, and controlling this in an educational portfolio format.

Following Miller's terminology of assessment ${ }^{23}$ (figure 1), the EURACT Educational Agenda and EUPA define competence as the capability to successfully perform discrete observational tasks in a defined assessment environment, in isolation from actual work. It includes the level of "knowing" (basic facts), "knowing how" (able to apply knowledge) and "showing how" (able to show skills) but it excludes the "doing" level, the performance in practice. Competency-based assessments measure what doctors do under examination conditions ${ }^{24}$. 
Performance is defined as the level of actual performance in clinical care and communication with patients in daily practice. It relates in the Miller terminology to the "doing" level. It is considered highly dependent on existing healthcare conditions and requirements, financial and structural opportunities, practice opportunities and support. Performance-based assessments measure what doctors do in practice.

Rethans and colleagues ${ }^{25}$ argue that Miller's triangle implicitly assumes that competence predicts performance, but that the exact relationship between competence and performance is much more complicated. Factors such as time pressure, day of the week, mood of patient and doctor or impact of the fore-going examination influence clinical performance just as does individual competence in specific domain-related knowledge areas. System-related influences include government programmes and initiatives, patient expectations, guidelines or policies developed by the practice facility, time, and accessibility to other health professionals. Individual-related influences cover the physical and mental health of the GP, their state of mind at the time of the performance assessment, and their relationship with others, including patients, peers, and their own family ${ }^{26}$. Therefore, contemporary assessment demands a more flexible, interactive figure than a pyramid, allowing a view of the performance of an individual doctor from different angels. Rethans et al. have suggested the inversion of Miller's pyramid, focussing on the top two tiers (figure 2):

Clearly, competence is an important prerequisite for performance, and is therefore represented in the triangle in the centre. But at least two further triangles (system-related and individual-related influences) carry performance on their shoulders, identifying performance as a product of many aspects. EUPA follows this complex model of performance. While it is quite reasonable to seek an association between competence and performance, it is unreasonable to expect it to be strong ${ }^{28,29}$.

The future of the discipline of general practice/family medicine depends on many factors ${ }^{30}$, but certainly these include the quality of actual daily performance of general practitioners in this field. The EURACT Educational Agenda ${ }^{31}$ has provided a comprehensive framework to teach the six core competencies outlined in the European definition in BME and ST and to monitor and assess the achievement of goals at the end of training. The Educational Agenda defines a well performing GP as a 


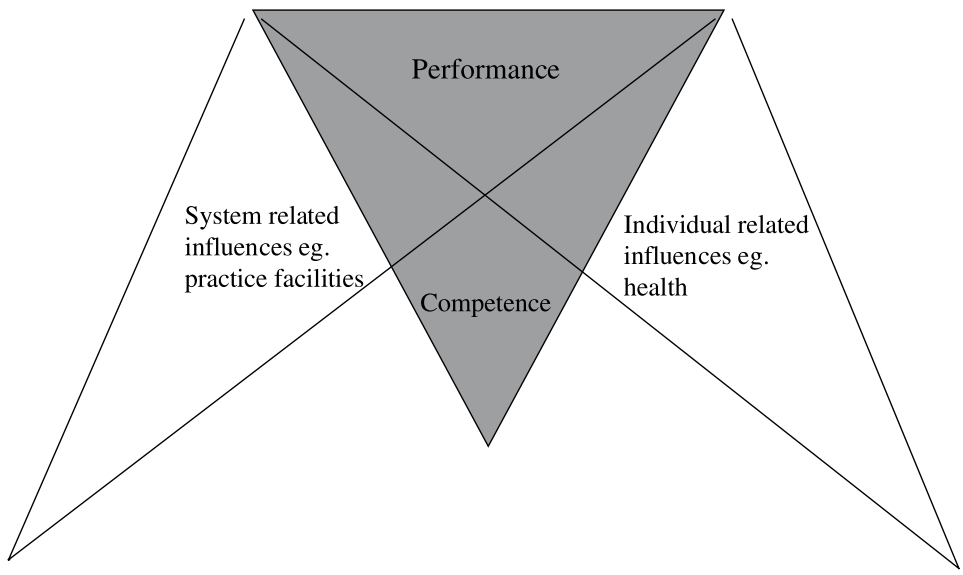

Figure 2: The Cambridge Model for delineating performance and competence ${ }^{27}$

professional which combines, uses and implements different elements of the professional skills in a fluid, normal and interiorized manner, knowing at every moment what are the background options and the key features related to it.

Now the questions remain: In actual practice, after gaining the competencies that are required to become a skilled exponent of the discipline and after finishing ST,

- Do general practitioners perform proper in regard to the six core competencies?

- Where, how and by whom can this be assessed?

- How and from whom can general practitioners get feedback on the identification of learning needs and on optimizing their daily performance?

To provide answers to these questions, EURACT has produced the present EURACT Performance Agenda (EUPA), being the third paper in a row following the European Definition of General Practice/Family Medicine by WONCA Europe in 2002, and the EURACT Educational Agenda in 2005. This document will close the loop between teaching knowledge, allowing students and trainees to gain competencies, and assessing actual performance of GPs in daily practice.

The first idea of EUPA was born in EURACT Council in 2007. Following an international study performed in Canada, Australia, New Zealand and the $\mathrm{UK}^{32}$, a task force conducted a survey asking all Council delegates from 38 nations 
about performance agendas or official documents in specialty training or continuing professional development in their countries. Council delegates from 34 countries responded. It was found that only a very few countries (e. g. UK, Israel) had a comparable document or policy on performance assessment in general practice/family medicine that went beyond a simple list of competencies. After a review of the literature, discussion in small groups involving all EURACT Council members took place over two years, following the chapters of the Educational Agenda and using the same terminology. Design of the document's outline, compilation of the final draft of the chapters focussing on the six core competencies and theoretical framing was done by a small group of authors. The final version of the document was approved by the EURACT Council in October 2013.

EUPA is a general, uniform and basic agenda of performance elements grouped in the six core competencies every general practitioner has to master in daily practice. Like the other two documents, it is supposed to be applicable and adaptable to different countries with different systems instead of being too normative. It deals with the process and result of actual work in daily practice, not with a teaching/learning situation, thus focussing on the CPD level, the longest phase of professional education (see chapter 3). It is concentrating on measurable/observable/assessable performance in general practice, applicable to various tasks (e.g. communication with patients, clinical routines, practice management etc) and in a wide range of settings. Thus, EUPA is not a list/directory outlining which competencies, skills and procedures are mandatory for general practice and which ones are optional.

Chapter 2 provides an overview of the six core competencies. Chapters 5 to 10 focus on each of the six. In every chapter, case vignettes of abilities in doctors' daily practice illustrate performance elements, their adequate assessment methods and the role of the assessor (tables 5-20).

The goal of such assessments is to see the whole picture of a practitioner's performance. A varied palette of methods is necessary to achieve this goal ${ }^{33}$. In general, assessment of a practitioner's performance can only be done on-site, at his/her workplace (workplace-based assessment; see chapter 11), preferably by direct observation. Performance assessment should refer to a theoretical framework (see chapter 4), should be organised in a programmatic way, i. e. embedded in daily practice routines, and cannot be improvised or organised as something "external" to continuing professional development (CPD) activities ${ }^{34,35}$. Ensur- 
ing the competent clinical performance of practicing doctors on an ongoing basis presents many challenges. The public, its elected representatives, employers, and doctors themselves may all have expectations and anxieties that need to be reconciled ${ }^{36,37}$. Because of the severe consequences an assessment of practice performance may have, it is essential that the procedure is both defensible to the stakeholders and fair in that it distinguishes well between good performers and underperformers ${ }^{38}$. Preferably, these programmes should remain in the hands of the profession, and the role of assessment should be a production tool of meaningful feedback to the individual GP.

The relation of performance assessment to quality management is discussed in chapter 3. The theoretical framework of the psychometrics and edumetrics of performance assessment is outlined in chapter 4.

A systematic review has observed six different suitable methods of evaluating performance: simulated patients (unannounced visits ${ }^{39}$ ); video observation; direct observation; on-site peer assessment; audit/review of medical records; and portfolio or appraisal. Evidence supporting improvement in routine practice is lacking so far ${ }^{40}$. Other authors have suggested other methods, e. g. feedback from patients, feedback from relatives of patients, and feedback from staff, or the use of checklists ${ }^{41,42,43,44}$.

Chapter 11 (glossary) provides more information about the above key performance assessment methods and defines other terms.

EUPA can help to shape various performance assessment activities held locally in general practice/family medicine, such as:

- Continuing professional development (CPD) cycles;

- Re-certification/re-accreditation/re-validation/re-licensing procedures;

- Peer hospitation programmes (e.g. visitatie programme ${ }^{45}$ );

- Practice audit programmes in quality management

and can give orientation for self assessment for reflective practitioners in their CPD.

It is hoped that the EURACT Performance Agenda EUPA will encourage European general practitioners to initialize performance agendas adapted to their national health system to further strengthen the role of general practice/family medicine in their country. 
